\title{
Correction to: On Slice Polyanalytic Functions of a Quaternionic Variable
}

\author{
Daniel Alpay, Kamal Diki, and Irene Sabadini(D
}

\section{Correction to: Results Math (2019) 74:17 https://doi.org/10.1007/s00025-018-0942-2}

In [2] we initiated the theory of polyanalytic functions of a quaternionic variable in the slice hyperholomorphic setting. While developing the associated functional calculus, see [1], and as it is discussed in [3, Remark 2.14], it turned out that Definition 3.1 in [2] is too general to obtain all the expected results valid in the slice hypercomplex setting. Thus, the slice polyanalytic functions of a quaternionic variable (or of a paravector variable, in the case of Clifford algebra-valued functions) have to be considered as a subclass of slice functions as in Definition 3.17 of [2].

The results presented in [2] for slice polyanalytic functions of a quaternionic variable stay valid when we use, instead of Definition 3.1 in [2], Definition 3.17 of the same paper.

Definition 1. (Slice polyanalytic functions) Let $M \in \mathbb{N}$ and denote by $\mathcal{C}^{M}(U)$ the set of continuously differentiable functions with all their derivatives up to order $M$ on an axially symmetric open set $U \subseteq \mathbb{H}$. We let $\mathcal{U}=\left\{(x, y) \in \mathbb{R}^{2}\right.$ : $x+I y \subset U\}$. A function $F: U \rightarrow \mathbb{H}$ is called a left slice function, if it is of the form

$$
F(q)=\alpha(x, y)+I \beta(x, y) \quad \text { for } q=x+I y \in U
$$

with the two functions $\alpha, \beta: \mathcal{U} \rightarrow \mathbb{H}$ that satisfy the compatibility conditions $\alpha(x,-y)=\alpha(x, y), \beta(x,-y)=-\beta(x, y)$. If in addition $\alpha$ and $\beta$ are in $\mathcal{C}^{M}(U)$

The original article can be found online at https://doi.org/10.1007/s00025-021-01364-y. 
and satisfy the poly Cauchy-Riemann equations of order $M \in \mathbb{N}$

$$
\frac{1}{2^{M}}\left(\partial_{x}+I \partial_{y}\right)^{M}(\alpha(x, y)+I \beta(x, y))=0, \quad \text { for all } I \in \mathbb{S}
$$

then $F$ is called left slice polyanalytic function of order $M \in \mathbb{N}$.

The definition is easily adapted in the case of right slice polyanalytic functions. Note that slice regular functions are obtained from the previous definition for $M=1$.

Using the Definition 1, all the results of slice polyanalytic functions that were investigated in [2] are valid in the quaternionic setting, possibly with some changes in the proofs and in the list below we discuss all of them in detail. Note that the open sets on which the slice polyanalytic functions are defined are necessarily axially symmetric.

(1) A slice function is said to be intrinsic when $\alpha, \beta$ in Definition 1 are realvalued. Two slice functions can be multiplied with the $*$-product, see [4]. Thus Proposition 3.3 and its proof are valid.

(2) Proposition 3.4, namely the Splitting Lemma, holds since its proof depends only on the splitting of the values of the functions. As a consequence also Proposition 3.5 holds since $f$ is clearly a slice function.

(3) The statement of Proposition 3.6 is valid but the proof follows the arguments used in the slice poly monogenic setting, see Theorem 3.7 of [1]. We point out that Theorem 2.16 in [3] also establishes the result.

The proof is as follows: let $f(q)=f(x+I y)=\alpha(x, y)+I \beta(x, y)$ be a left slice polyanalytic function of order $N$. By fixing a basis $1, e_{1}, e_{2}, e_{1} e_{2}$ of $\mathbb{H}$, and setting $e_{0}=1, e_{3}=e_{1} e_{2}$, we have $\alpha=\sum_{\ell=0}^{3} \alpha_{\ell} e_{\ell}, \beta=\sum_{\ell=0}^{3} \beta_{\ell} e_{\ell}$, where the functions $\alpha_{\ell}, \beta_{\ell}$ are real-valued and are, respectively, even and odd in the second variable. Since the basis elements $e_{\ell}$ are linearly independent, the system expressing the slice polyanalyticity can be rewritten in terms of the components of $f$, in other words, each $\mathbb{C}_{I}$-valued function $F_{\ell}=\alpha_{\ell}+I \beta_{\ell}$ is polyanalytic and $\overline{F_{\ell}(x-I y)}=F_{\ell}(x+I y)$. By the classical result applied to each function $F_{\ell}$, we have $F_{\ell}(x+I y)=\sum_{k=0}^{N-1}(x-I y)^{k} f_{k, \ell}(x+I y)$ where the functions $f_{k, \ell}$ are $\mathbb{C}_{I}$-valued and satisfy the Cauchy-Riemann system. Since $\overline{F_{\ell}(x-I y)}=\sum_{k=0}^{N-1}(x-I y)^{k} \overline{f_{k, \ell}(x-I y)}=\sum_{k=0}^{N-1}(x-I y)^{k} f_{k, \ell}(x+I y)$ we have $\overline{f_{k, \ell}(x-I y)}=f_{k, \ell}(x+I y)$ and so each $f_{k, \ell}$ is a slice function, as its real components satisfy the even-odd conditions. We then deduce

$$
\begin{aligned}
f(x+I y) & =\sum_{\ell=0}^{3} F_{\ell}(x+I y) e_{\ell}=\sum_{\ell=0}^{3} \sum_{k=0}^{N-1}(x-I y)^{k} f_{k, \ell}(x+I y) e_{\ell} \\
& =\sum_{k=0}^{N-1}(x-I y)^{k} f_{k}(x+I y), \quad f_{k}(x+I y)=\sum_{\ell=0}^{3} f_{k, \ell}(x+I y) e_{\ell},
\end{aligned}
$$

where the functions $f_{k}$ are evidently left slice regular, and this concludes the proof. 
We note that Corollary 3.7 and its proof remains the same.

(4) Theorem 3.8 in [2] remains valid, but in the proof the last five lines have to substituted by the observation that since $f, g$ are slice function, and since $f_{I}=g_{I}$, i.e. they coincide on $\Omega_{I}$, then they coincide on $\Omega$, by Proposition 5 in [4].

(5) The representation formula in Theorem 3.9 is valid but there is no need of a proof since it is a consequence of the sliceness of a slice polyanalytic function, see [4]. Corollary 3.11 and 3.12 are correct but trivial.

(6) The first part of Proposition 3.13 is not anymore needed so the statement become as below; the proof remains valid.

Let $\Omega_{I}$ be a domain in $\mathbb{C}_{I}$ symmetric with respect to the real axis and such that $\Omega_{I} \cap \mathbb{R} \neq \emptyset$. Let

$$
f(z)=\sum_{k=0}^{N-1} \bar{z}^{k} h_{k}(z)
$$

where $h_{k}: \Omega_{I} \longrightarrow \mathbb{H}$ are holomorphic functions such that $\overline{h_{k}(\bar{z})}=h_{k}(z)$. Then the unique slice polyanalytic extension of $f$ is

$$
\operatorname{ext}(f)(q):=\sum_{k=0}^{N-1} \bar{q}^{k} \operatorname{ext}\left(h_{k}\right)(q) ; \forall q=x+I_{q} y \in \Omega .
$$

(7) In the sections 4, 5 of [2] concerning the function spaces, in order to have kernels that are slice functions we use the $*$-product of (left) slice functions in the first variable, and we write the kernels in Theorems 4.6, Theorem 5.2 and Proposition 5.3 as $K_{N}(q, r)=e_{*}(q \bar{r}) * \varphi_{N}(q, r)$ and $B_{S}^{N}(q, r)=$ $P_{N}(q, r) Q_{N}(q, r) * \psi_{N}(q, r)=R_{N}(q, r) L_{N}(q, r) * \psi_{N}(q, r)$ where the slice functions (not necessarily real-valued) $\varphi_{N}, \psi_{N}$ are given by

$$
\begin{aligned}
\varphi_{N}(q, r)= & \sum_{k=0}^{N-1}(-1)^{k}\left(\begin{array}{c}
N \\
k+1
\end{array}\right) \frac{1}{k !}(\bar{q} q-q \bar{r}-\bar{q} r+\bar{r} r)^{k *} \\
\psi_{N}(q, r)= & \sum_{k=0}^{N-1}(-1)^{k}\left(\begin{array}{c}
N \\
k+1
\end{array}\right)\left(\begin{array}{c}
N+k \\
N
\end{array}\right)(1-\bar{q} r-q \bar{r}+\bar{q} q \bar{r} r)^{(N-1-k) *} \\
& *(\bar{q} q-q \bar{r}-\bar{q} r+\bar{r} r)^{k *}
\end{aligned}
$$

Data availability Not applicable.

\section{References}

[1] Alpay, D., Colombo, F., Diki, K., Sabadini, I.: Poly slice monogenic functions, Cauchy formulas and the $P S$-functional calculus. arXiv:2011.13912 (2020)

[2] Alpay, D., Diki, K., Sabadini, I.: On slice polyanalytic functions of a quaternionic variable. Results Math. 74(1), 17 (2019) 
[3] Ghiloni, R.: Slice-by-slice and global smoothness of slice regular and polyanalytic functions. arXiv:2011.09919 (2020)

[4] Ghiloni, R., Perotti, A.: Slice regular functions on real alternative algebras. Adv. Math. 226, 1662-1691 (2011)

Daniel Alpay

Schmid College of Science and Technology

Chapman University

Orange CA92866

USA

e-mail: alpay@chapman.edu

Kamal Diki and Irene Sabadini

Dipartimento di Matematica

Politecnico di Milano

Via E. Bonardi, 9

20133 Milano

Italy

e-mail: kamal.diki@polimi.it;

irene.sabadini@polimi.it

Publisher's Note Springer Nature remains neutral with regard to jurisdictional claims in published maps and institutional affiliations. 